\title{
Case Report: Visceral Leishmaniasis and Hemophagocytic Lymphohistiocytosis: Three Clinical Cases, Three Different Patterns
}

\author{
Geydson Silveira Cruz, ${ }^{1,2,3 \star}$ Amélia Ribeiro de Jesus, ${ }^{2,3}$ Roque Pacheco Almeida, ${ }^{2,3}$ and Maria Aurélia Porto ${ }^{1}$ \\ ${ }^{1}$ Hematology Service and Transfusional Unit, University Hospital-Universidade Federal de Sergipe, Aracaju, Sergipe, Brazil; ${ }^{2}$ Molecular Biology \\ and Immunology Laboratory, University Hospital_Universidade Federal de Sergipe, Aracaju, Sergipe, Brazil; ${ }^{3}$ Health Sciences Post Graduate
} Program-Universidade Federal de Sergipe, Aracaju, Sergipe, Brazil

\begin{abstract}
Visceral leishmaniasis $(\mathrm{VL})$ is a neglected tropical disease with more than 30,000 cases annually reported worldwide. In Brazil, about 3,700 cases are annually reported. The VL clinical presentation is variable, from asymptomatic to severe cases with a high risk of death. We reported three cases of VL with clinical sign similarities but distinct development. All cases had bone marrow hemophagocytosis and hemophagocytic lymphohistiocytosis (HLH) criteria. $\mathrm{HLH}$ is a rare condition that may have secondary causes, including infectious and parasitic diseases, like VL. The delayed recognition of the secondary $\mathrm{HLH}(\mathrm{sHLH}$ ) association to VL may cause unfavorable outcomes and death.
\end{abstract}

\section{INTRODUCTION}

Visceral leishmaniasis (VL) is a neglected, poverty-related, vector-borne parasitic disease of tropical climates. The World Health Organization (WHO) reported more than 30,000 new VL cases in high-burden countries in $2014 .{ }^{1}$ More than $90 \%$ of the VL cases in the world are present in seven countries (including Brazil). Visceral leishmaniasis cases from Brazil are responsible for more than $99 \%$ of Latin America's reported cases. ${ }^{2}$ In Brazil, leishmania infantum causes VL, and the endemic covers 21 out of 26 states. Between 2008 and 2018, the official notification system reported 41,490 cases, about 3,700 cases annually; $53(1 \%)$ of them were from northeastern Brazil. $^{3}$

The clinical presentation of leishmaniasis cases varies. The person can be asymptomatic (the "subclinical" form), with signs of fever, malaise, and little hepatosplenomegaly; and later, the person converts to positive in a delayed-type hypersensitivity test (Montenegro test), or the person can present with classical visceral leishmaniasis (VL). Fever, pancytopenia, and splenomegaly are common clinical manifestations of classical VL, and the patient can present complications that include bleeding and secondary bacterial infection. VL is the most severe form of leishmaniasis and may cause death if the diagnosis and treatment are delayed and the patient is not adequately managed. ${ }^{4}$ Atypical symptoms can occur in immunocompromised patients or in highly endemic areas. The diagnostic is confirmed by direct parasitic visualization or by culture from bone marrow, spleen, or lymph node. Recombinant antigen (rK) 39 is a fast and cheap immunochromatographic, highly sensitive test commonly used for diagnosis. Molecular tests, like polymerase chain reaction (PCR) tests, are useful, and these tests are the most sensitive available. $^{5}$

Hemophagocytic lymphohistiocytosis $(\mathrm{HLH})$ is a rare condition that may have secondary causes. HLH has both infectious and noninfectious triggers. Secondary HLH (sHLA) cases are associated more commonly to viral infections, but other infectious agents were reported: bacteria,

*Address correspondence to Geydson Silveira Cruz, Hospital Universitário de Sergipe-Universidade Federal de Sergipe (UFS), Unidade Transfusional, Rua Cláudio Batista, 505 Palestina, Aracaju, Sergipe, Brazil 49.060-025. E-mail: geydson.cruz@ebserh.gov.br including Mycobacteria tuberculosis; fungus, including Histoplasma; and parasites, such as Toxoplasma, Plasmodium, and Leishmania. ${ }^{6-8}$ This article aims to describe three clinical cases of visceral leishmaniasis with three different patterns of $\mathrm{HLH}$ development. All patients gave their consent to participate in the report.

\section{CASE REPORTS}

Case 1. We admitted to our hospital a 26-year-old man, a gardener at a local park, who had fever and jaundice. The onset of his symptoms was 40 days before hospitalization. Before this last hospitalization, the patient was in another hospital, where he received treatment of leptospirosis. Because the laboratory exams did not confirm a leptospirosis diagnosis, the patient came to our hospital for specialized evaluation. He had fever, jaundice, bleeding, and dyspnea. In the physical examination, cutaneous-mucous bleeding and splenomegaly stood out. We did not find previous comorbidities. On the first day of hospitalization, the patient worsened, and we referred him to the intensive care unit. He tested negative to HIV, hepatitis $B$, and hepatitis $C$. Peripheral blood count evidenced anemia and severe thrombocytopenia (Table 1). Coagulopathy and liver enzymes elevation was also present. He also tested positive for the rK39 antigen. We performed a bone marrow aspiration, which confirmed the VL diagnosis. There were few amastigotes forms and evident hemophagocytosis. Then, we performed a liposomal amphotericin B administration. Two days later, the patient died of multiple organ failure.

Case 2. A 20-year-old woman who transferred from another hospital came to ours because she had no improvement in her symptoms, and some tests suggested VL. The onset of her symptoms was a month before admission to our hospital. She had a fever, hepatosplenomegaly, choluria, and pancytopenia. Therefore, she received treatment with an antimicrobial, cefepime, and metronidazole. Table 1 presents laboratory data. The patient tested negative for HIV, hepatitis $B$, and hepatitis $C$ and tested positive for the rK39 antigen. Bone marrow aspiration did not show the presence of amastigotes. There was significant hemophagocytosis, with no evidence of hematological neoplasia.

On the third day of hospitalization, she worsened and went to the intensive care unit. There was evidence of liver failure 
TABLE 1

Laboratory data of the patients

\begin{tabular}{|c|c|c|c|}
\hline & Patient 1 & Patient 2 & Patient 3 \\
\hline Hemoglobin (g/dL) & 9.6 & 8.4 & 7.5 \\
\hline Hematocrit (\%) & 29.2 & 24.6 & 21.2 \\
\hline White blood cell count $\left(/ \mathrm{mm}^{3}\right)$ & 7,450 & 410 & 2,620 \\
\hline Neutrophil $\left(/ \mathrm{mm}^{3}\right)$ & 1,490 & 123 & 1,126 \\
\hline Platelets $\left(/ \mathrm{mm}^{3}\right)$ & 19,200 & 37,300 & 85,400 \\
\hline International normalized ratio (INR) & 3.2 & 4.9 & 1,3 \\
\hline Fibrinogen & 91.6 & NA & NA \\
\hline $\begin{array}{l}\text { Aspartate transaminase, units per liter } \\
\text { (AST U/L) }\end{array}$ & 1,072 & 243 & 329 \\
\hline $\begin{array}{l}\text { Alanine transaminase, units per liter (ALT } \\
\text { U/L) }\end{array}$ & 1,838 & 124 & 221 \\
\hline Creatinine $(\mathrm{mg} / \mathrm{dL})$ & 2.9 & 0.4 & 0.7 \\
\hline Urea (mg/dL) & 53.0 & 37.0 & 99 \\
\hline Ferritin $(\mathrm{ng} / \mathrm{mL})$ & NA & $10,770.9$ & 577 \\
\hline Bilirubin (mg/dL) & 22.6 & 27.4 & 5.6 \\
\hline Albumin (g/dL) & 1.6 & 3.9 & 2.3 \\
\hline Globulin (g/dL) & 4.2 & 2.4 & 9.0 \\
\hline Triglycerides (mg/dL) & NA & 225 & NA \\
\hline $\begin{array}{l}\text { Lactate dehydrogenase, units per liter } \\
\text { (LDH U/L) }\end{array}$ & 821 & 412 & 299 \\
\hline Bone marrow hemophagocytosis & Yes & Yes & Yes \\
\hline Bone marrow amastigotes & Yes & No & Yes \\
\hline $\begin{array}{l}\text { Bone marrow culture, Novy-MacNeal- } \\
\text { Nicolle medium (NNN; for } \\
\text { leishmaniasis) }\end{array}$ & Positive & Positive & Positive \\
\hline
\end{tabular}

with bleeding at puncture sites and mucous membranes. We decided to expand the antimicrobial spectrum with the administration of meropenem and vancomycin. The hepatology team indicated liver transplantation because of liver failure without improvement. Hematology evaluation suggested treatment of sHLH. Therefore, the administration of methylprednisolone (1 $\mathrm{g} /$ day for 3 consecutive days) and human immunoglobulin ( $1 \mathrm{~g} / \mathrm{Kg}$ for 2 consecutive days) was necessary. We also used liposomal amphotericin $B$ to treat the patient for the cause of HLH (VL).

During therapy, there was clinical worsening with respiratory failure; there was a need for orotracheal intubation and mechanical ventilation. We treated renal failure with dialysis, and after the end of pulse therapy, we maintained corticosteroids at a dose of $1 \mathrm{mg} / \mathrm{kg}$ of prednisolone. After 7 days of treatment for VL, the patient was removed from artificial ventilation and discharged on day 8 from the ICU. In the inpatient unit, her clinical condition continued to improve, with discharge on the day 28 of hospitalization.

Case 3. We admitted a 41-year-old woman at our hospital from the outpatient unit. The patient had a clinical picture of fever, weight loss, and increased abdominal volume. Symptoms started about 15 days before hospitalization. After admission, she also presented a cough and jaundice. Clinical examination showed splenomegaly.

Table 1 shows laboratory tests. Serologies for HIV, hepatitis $B$, and hepatitis $C$ were all negative. Bone marrow aspiration showed few amastigote forms, with remarkable hemophagocytosis. Therapy with liposomal amphotericin B was introduced for 5 days, as guided by national protocols, associated with $1 \mathrm{mg} / \mathrm{kg} / \mathrm{d}$ of prednisone. With the progressive improvement of symptoms, such as fever, and an increase in peripheral blood cell count, we discharged the patient on day 17 of hospitalization. The corticosteroid therapy was gradually tapered in 30 days.
The two patients who did not die received regular posttreatment follow-up for 1 year and are clinically well.

\section{DISCUSSION}

In this article, we showed how VL has different patterns of development, even showing similarity among the cases. We hospitalized all patients diagnosed with VL, cytopenias in peripheral blood, fever, splenomegaly, and jaundice. VL diagnosis was based on the clinical criteria and confirmed by tests such as rK39, parasite visualization by bone marrow aspiration, and culture for Leishmania. On the other hand, problems such as the presence of hypofibrinogenemia with liver disfunction and highlevel ferritin dosage are not common in patients with this condition. Because of that, we needed to raise the possibility of additional diagnosis. In the bone marrow evaluation, including the collection of culture for Leishmania sp., we identified hemophagocytosis in all samples collected. Despite the absence of amastigotes in the second patient's results, the bone marrow culture confirmed the diagnostic hypothesis of VL.

With the finding of bone marrow hemophagocytosis, we remembered the hypothesis of SHLH to VL. This condition causes inflammatory cytokine release and macrophagic activation, and these problems lead to systemic inflammatory signs and symptoms. We can classify HLH as primary or secondary. Genetic mutations cause primary $\mathrm{HLH}$, and this condition commonly appears in infancy. On the other hand, the secondary one, or reactive HLH (sHLH), is the most common form in adults. The reactive HLH is generally triggered by infectious agents, like the Epstein-Barr virus, in a previous immune dysfunction, like a hematologic malignancy. The hyperinflammatory profile seems to have most relevant role rather than cytotoxicity dysfunction in $\mathrm{SHLH}^{7,9,10}$ 
Genetic defects in proteins involved in the cytotoxic function of $\mathrm{T}$ and NK lymphocytes cause familial (primary) $\mathrm{HLH}$ (fHLH). It is an autosomal recessive disease that appears in 1 out of 50,000 live-born infants. Viral infections are the most common triggers of $\mathrm{HLH}$. Cases of sHLH commonly occur in adults, whose defects of T and NK lymphocytes are acquired. Generally, there is an underlying infectious disease, an autoimmune pathology, or even a malignant disorder that leads to uncontrolled activation of the immune system. However, in some situations, the triggering cause is not identified. ${ }^{11}$ The diagnosis is performed based on $\mathrm{HLH}-$ 2004 diagnostic criteria. $^{12}$ Patients present signs and symptoms like fever, organomegaly, liver dysfunction, coagulopathy, cytopenia, and so on. Commonly, health professionals perform other laboratory tests in $\mathrm{HLH}$, despite not being part of the diagnostic criteria. We found transaminitis in all patients, and there are reports of the presence of this condition in $83.6 \%$ of cases.

Coagulopathy and hypoalbuminemia are also reported. ${ }^{13}$ Severe organic deterioration and life-threatened diseases may arise, so patients must receive therapy immediately. Otherwise, they may die. The delay for the right diagnosis of the first patient, in addition to the confusion caused by the symptoms that motivated the initial treatment of leptospirosis, may have contributed to the severe systemic impairment and reduced possibility of a better outcome for case 1.

As previously reported, $\mathrm{HLH}$ has both infectious and noninfectious triggers, and the viruses are the most reported infectious ones. However, bacterial infections, including tuberculosis, are described as causing HLH. Parasitic and fungal infections are less related to HLH triggers, but there are reports of secondary occurrences to Histoplasma, Toxoplasma, Plasmodium, and Leishmania. ${ }^{6-8}$ Currently, HLH and NK cell dysfunction have also been cited as possible clinical conditions in the context of COVID-19 infection, disease that has also been associated with a cytokine storm. ${ }^{14,15}$

There was no availability of SCD25 (sIL-2Ra) in the reported cases, which would contribute significantly to the diagnostic confirmation of HLH. On the other hand, the information that patients with VL can express this marker ${ }^{16}$ reinforces the need to evaluate them by including this analysis in their clinical approach. Another factor to be assessed is whether the presence of high levels of SCD25 (sIL-2Ra) in patients with more severe VL could be the cases in which they have SHLH to VL. Despite having high mortality, cases of sHLH to VL (as well as other infectious pathologies), may have a better outcome with the appropriate treatment. ${ }^{17-19}$

Importantly, IVIg is often used for the treatment of $\mathrm{HLH}$, which is used along with steroids in patients with active infection. ${ }^{10}$ However there are other treatment options for $\mathrm{HLH}$, such as etoposide. None of the patients in this series required treatment with etoposide, but in the second case reported, the possibility of using etoposide was consideredhowever we opted to treat the underlying disease, VL, associated with high-dose immunoglobulin and costicosteroids. With the progressive clinical improvement, the possibility of using chemotherapy was ruled out. The treatment of VL downregulates the cytokine storm within 7 days, normalizing the serum levels of the cytokines after 28 days of follow-up. ${ }^{16}$ Clinicians are understandably reluctant to give etoposidebased treatment to patients with active infections. There are two new agents holding great promise for treating severe infection associated HLH: JAK inhibition (ruxolitinib) ${ }^{20}$ and IFN gamma inhibition (emapalumab). ${ }^{10,21}$

In conclusion, VL is a potentially severe condition that may cause death if not recognized promptly and treated appropriately. We must assess severe cases of VL carefully, as $\mathrm{SHLH}$ can be a consequence of $\mathrm{VL}$, a possibility that strengthens the importance of early identification and establishment of treatment of this infectious pathology and inflammatory condition.

Received November 6, 2020. Accepted for publication March 23, 2021.

Published online May 24, 2021.

Authors' addresses: Geydson Silveira Cruz, Hematology Service and Transfusional Unit, University Hospital-Universidade Federal de Sergipe, Aracaju, Sergipe, Brazil, Molecular Biology and Immunology Laboratory, University Hospital-Universidade Federal de Sergipe, Aracaju, Sergipe, Brazil, and Health Sciences Post Graduate Program-Universidade Federal de Sergipe, Aracaju, Sergipe, Brazil, E-mail: geydson@hotmail.com. Amélia Ribeiro de Jesus and Roque Pacheco Almeida, Molecular Biology and Immunology Laboratory, University Hospital—Universidade Federal de Sergipe, Aracaju, Sergipe, Brazil, and Health Sciences Post Graduate Program-Universidade Federal de Sergipe, Aracaju, Sergipe, Brazil, E-mails: ameliaribeirodejesus@ gmail.com and roquepachecoalmeida@gmail.com. Maria Aurélia Porto, Hematology Service and Transfusional Unit, University HospitalUniversidade Federal de Sergipe, Aracaju, Sergipe, Brazil, E-mail: maria.porto281@gmail.com.

\section{REFERENCES}

1. Focus E, 2016. WHO, Weekly epidemiological record: leishmaniasis in high-burden countries, an epidemiological update based on data reported in 2014. Wkly Epidemiol Rec / Heal Sect Secr Leag Nations 91: 287-296.

2. Pan American Health Organization-PAHO, 2019. Leishmaniasis: Epidemiological Report in the Americas. Available at: http:// iris.paho.org/xmlui/handle/123456789/50505\%0D. Accessed October 2019.

3. BRASIL M da S, 2019. LEISHMANIOSE VISCERAL-Casos confirmados notificados no Sistema de Informação de Agravos de Notificação. DATASUS—SINAN. Available at: http:// tabnet.datasus.gov.br/cgi/tabcgi.exe?sinannet/cnv/ leishvbr.def. Accessed January 16, 2019.

4. Burza S, Croft SL, Boelaert M, Maxfield L, Crane JS, 2018. Leishmaniasis. Lancet 392: 951-970.

5. Aronson N, Herwaldt BL, Libman M, Pearson R, Lopez-Velez R, Weina P, Carvalho E, Ephros M, Jeronimo S, Magill A, 2017. Diagnosis and treatment of leishmaniasis: Clinical Practice Guidelines by the Infectious Diseases Society of America (IDSA) and the American Society of Tropical Medicine and Hygiene (ASTMH). Am J Trop Med Hyg 96: 24-45.

6. Diamantidis MD, Palioura A, loannou M, Tsangalas E, Karakousis $\mathrm{K}, 2020$. Hemophagocytic lymphohistiocytosis as a manifestation of underlying visceral leishmaniasis. Cureus 12: 4-8.

7. Al-Samkari H, Berliner N, 2018. Hemophagocytic lymphohistiocytosis. Annu Rev Pathol Mech Dis 13: 27-49.

8. Koubâa M, Mâaloul I, Marrakchi C, Mdhaffar M, Lahiani D, Hammami B, Makni F, Ayedi A, Ben Jemâa M, 2012. Hemophagocytic syndrome associated with visceral leishmaniasis in an immunocompetent adult-case report and review of the literature. Ann Hematol 91: 1143-1145.

9. Carvelli $J$ et al., 2020. Functional and genetic testing in adults with $\mathrm{HLH}$ reveals an inflammatory profile rather than a cytotoxicity defect. Blood 136: 542-552.

10. La Rosée $P$ et al., 2019. Recommendations for the management of hemophagocytic lymphohistiocytosis in adults. Blood 133: 2465-2477.

11. Hayden A, Park S, Giustini D, Lee AYY, Chen LYC, 2016. Hemophagocytic syndromes (HPSs) including hemophagocytic 
lymphohistiocytosis (HLH) in adults: a systematic scoping review. Blood Rev 30: 411-420.

12. Allen CE, McClain KL, 2015. Pathophysiology and epidemiology of hemophagocytic lymphohistiocytosis. Hematology 2015: 177-182.

13. Otrock ZK, Eby CS, 2015. Clinical characteristics, prognostic factors, and outcomes of adult patients with hemophagocytic lymphohistiocytosis. Am J Hematol 90: 220-224.

14. Que $Y$ et al., 2021. Cytokine release syndrome in COVID-19: a major mechanism of morbidity and mortality. Int Rev Immunol 22: 1-14.

15. van Eeden C, Khan L, Osman MS, Tervaert JWC, 2020. Natural killer cell dysfunction and its role in COVID-19. Int J Mol Sci 21: $1-17$.

16. Duthie MS et al., 2014. Alteration of the serum biomarker profiles of visceral leishmaniasis during treatment. Eur J Clin Microbiol Infect Dis 33: 639-649.
17. Hayden A, Lin M, Park S, Pudek M, Schneider M, Jordan MB, Mattman A, Chen LYC, 2017. Soluble interleukin-2 receptor is a sensitive diagnostic test in adult HLH. Blood Adv 1:2529-2534.

18. La Rosee $P, 2015$. Treatment of hemophagocytic lymphohistiocytosis in adults. Hematology 2015: 190-196.

19. Lerolle $\mathrm{N}$ et al., 2016. Diversity and combinations of infectious agents in 38 adults with an infection-triggered reactive haemophagocytic syndrome: a multicenter study. Clin Microbiol Infect 22: 268.e1-268.e8.

20. Ahmed A et al., 2019. Ruxolitinib in adult patients with secondary haemophagocytic lymphohistiocytosis: an open-label, singlecentre, pilot trial. Lancet Haematol 6: e630-e637.

21. Locatelli $F$ et al., 2018. Safety and efficacy of Emapalumab in pediatric patients with primary hemophagocytic lymphohistiocytosis. Blood 132 (Suppl 1): LBA-6. 